\title{
The Influence of Tinnitus on the Audiometric Threshold of Sufferers
}

\author{
Onyinye Ukaegbe ${ }^{1}$ Basil Ezeanolue $^{1}$ Foster Orji $^{1}$ \\ ${ }^{1}$ Department of Otorhinolaryngology, University of Nigeria Teaching \\ Hospital, Ituku-Ozalla Enugu, Enugu, Nigeria \\ Int Arch Otorhinolaryngol 2016;20:339-343.

\begin{abstract}
Address for correspondence Onyinye Ukaegbe, FWACS, MSc Audiology for ENT Practice, MBBS, Department of Ozalla Enugu, Enugu, Nigeria (e-mail: onyikasi@yahoo.com).
\end{abstract} \\ Otorhinolaryngology, University of Nigeria Teaching Hospital, Ituku-
}

\begin{abstract}
Introduction Tinnitus is a worldwide problem.

Objective The objective of this study is to evaluate the audiometric hearing thresholds of adult patients with ongoing tinnitus as their only otological symptom.

Methods We evaluated the hearing thresholds of 43 adult patients with ongoing tinnitus and no history of hearing loss from the otolaryngology department of a tertiary health institution at speech and high frequencies. A total of 56 tinnitus ears were compared against 30 contralateral normal ears as well as with the 100 ears of 50 healthy volunteers.

Results The study group consisted of 11 (25.6\%) males and 32 (74.4\%) females with a mean age of $40.9 \pm 11.7$. The mean Pure Tone Average of the 56 tinnitus ears was $14.8 \pm 9$, while that of the 100 control ears was $11.2 \pm 6(U=2078, p=0.008)$. The mean pure tone average of the control was also significantly lower than that of the 30 contralateral normal ears of the tinnitus sufferers $(U=1136, p=0.02)$. We observed mild to moderate hearing loss in $10(23 \%)$ of the participants. We observed no hearing loss among the control group.

Keywords

- tinnitus

- hearing threshold

- audiometry

Conclusion A proportion of tinnitus sufferers with self-professed normal hearing are likely to have mildly elevated pure tone audiometric thresholds. In patients with unilateral tinnitus, such elevated pure tone hearing thresholds are likely to be in the tinnitus ear and the contralateral non-tinnitus ear.
\end{abstract}

\section{Introduction}

Tinnitus is a worldwide problem and is estimated to affect $12 \%$ to $15 \%$ of the adult population, with a male preponderance. ${ }^{1}$ Studies indicate that approximately one third of the global population will experience tinnitus in their lifetime, but tinnitus as a symptom remains a challenge to most clinicians. ${ }^{2,3}$

Tinnitus is usually described as a buzzing, ringing, humming, clicking, blowing or drum-like sound and involves the perception of pure tones or frequency ranges of noise similar to white noise with no particular pattern of organization. ${ }^{4,5}$

As much as $80 \%$ of patients seen in the otorhinolaryngology clinic may have tinnitus, and it has been associated with a variety of ear pathologies among which hearing loss remains the most common. ${ }^{3,6-9}$ Tinnitus has been cited as a potentially debilitating symptom and can be a source of distress to the affected individual especially in cases where there is an associated hearing loss. ${ }^{3,10,11}$

Tinnitus is believed to be generated from several disorders of the physiological mechanism of hearing. ${ }^{12}$ These disorders are believed to exist in the peripheral sensorineural activity of the peripheral auditory system or in the central neural pathways. ${ }^{12}$ Tinnitus may also arise from the interaction between a defective peripheral input and a dysfunctional central neural pathway. ${ }^{12}$

The progress in tinnitus management has been slow especially in developing countries like Nigeria and there is a paucity of data on the hearing thresholds of tinnitus patients with self-professed normal hearing and no received

September 6, 2015

accepted

October 23, 2015

published online

February 15, 2016
Dol http://dx.doi.org/

10.1055/s-0035-1571271. ISSN 1809-9777.
Copyright (c) 2015 by Thieme Publicações License terms Ltda, Rio de Janeiro, Brazil

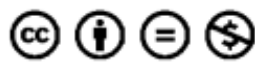


additional otological symptoms, probably due to the fact that most of these patients may not present to otorhinolaryngologists. It is important to determine the pure tone audiometric hearing thresholds of these patients who present with tinnitus as their only otological symptom, to find out how it defers from that of healthy controls, as well as to evaluate the hearing thresholds in the contralateral non-tinnitus ears of patients with unilateral tinnitus for better counselling of the patient and overall management of the tinnitus patient.

\section{Materials and Methods}

We conducted the study in the outpatient otorhinolaryngology clinics of a tertiary hospital over a year period ending in May 2014. We obtained approval from the Ethics review committee of our institution and informed consent from each participant.

We recruited for the study forty-three consecutive patients aged 21 - 58 years with a mean age of $40.9 \pm 11.7$ who had self-reported normal hearing and ongoing tinnitus as their only otorhinolaryngology complaint. Thirteen (30.2\%) of the patients had bilateral tinnitus, thereby making a total of 56 ears with ongoing tinnitus available for analysis, while 30 contralateral ears were symptom free in the 30 (69.8\%) unilateral tinnitus sufferers. We excluded subjects from the study if they had a prior history of hearing loss, or had any debilitating illness. The average duration of tinnitus was 17.3 months \pm 20.3 . Persistent tinnitus was observed in 20 (46.5\%) of the participants while 23 (53.5\%) had intermittent tinnitus. We obtained a history of noise exposure from 8 (18.6\%) of the participants, while 14 (32.6\%) of the participants had been exposed to drugs likely to cause tinnitus. Two of the subjects were diabetic, one was hypertensive and diabetic, seven of the subjects were hypertensive, and all were on medication.

The control group consisted of 50 healthy volunteers (100 ears) aged 21 to 57 years with a mean of $38.7 \pm 9.7$ years, who were pooled from the hospital staff, as well as the medical and nursing students of the institution. Eighteen (36\%) were male and 32 (64\%) were female. A comprehensive history and detailed ear, nose, and throat examination was performed in both groups.

\section{Pure Tone Audiometry}

All the participants in the study and control group underwent pure tone audiometry using an Amplivox 260 diagnostic audiometer (Amplivox Limited, Oxforshire, England).
Tests were conducted in a sound proof booth and using circumaural head phones. We assessed the frequencies of $500 \mathrm{~Hz}, 1000 \mathrm{~Hz}, 2000 \mathrm{~Hz}, 3000 \mathrm{~Hz}, 4000 \mathrm{~Hz}, 6000 \mathrm{~Hz}$, and $8000 \mathrm{~Hz}$ for the air conduction thresholds while $500 \mathrm{~Hz}$, $1000 \mathrm{~Hz}, 2000 \mathrm{~Hz}$, and $4000 \mathrm{~Hz}$ were assessed for the bone conduction thresholds with the use of a bone vibrator placed on the appropriate mastoid process. The three standard rules of masking were applied. We calculated the Pure Tone Average (PTAv) from the average of the four air conduction speech frequencies of $500 \mathrm{~Hz}, 1000 \mathrm{~Hz}$, $2000 \mathrm{~Hz}$, and $4000 \mathrm{~Hz}$. We calculated the high frequency pure tone average from the average of $6 \mathrm{KHz}$ and $8 \mathrm{KHz}$. Audiometric results were graded as: normal hearing ( $\leq 25 \mathrm{dBHL}$ ), mild hearing loss (26-40 dBHL), moderate hearing loss (41-60 dBHL), severe hearing loss (61-80 $\mathrm{dBHL})$, and profound hearing loss $(\geq 81 \mathrm{dBHL}){ }^{13}$ The hearing loss was classified as "Sensorineural" if both the air and bone conduction thresholds were above $25 \mathrm{dBHL}$ or "Conductive" in cases with air-bone gaps of $10 \mathrm{dBHL}$ or more or "Mixed" if both the air and bone conduction thresholds were above $25 \mathrm{dBHL}$ with air-bone gaps of 10 dBHL or more. ${ }^{14}$

We used the Statistical Package for Social Sciences version 15 (IBM, Armonk, U.S.A.) in the statistical analysis. We applied the independent samples $t$-test and Mann Whitney $U$ test in comparing the mean pure tone thresholds for the 56 tinnitus ears, 30 non-tinnitus ears, and the 100 control ears. The results are single-tailed and the significance level was set at less than 0.05 .

\section{Results}

-Table 1 outlines the age and gender distribution of the participants. The differences in the mean age between the study group and the control, as well as their gender distributions, were not significant ( $p=0.3$ and 0.1 , respectively).

We observed bilateral tinnitus in 13 (30.2\%) of subjects. Fourteen (32.6\%) had tinnitus in their right ear only, while 16 (37.2\%) of the subjects had tinnitus in the left ear only.

\section{Hearing Threshold of $\mathbf{4 3}$ Tinnitus Sufferers and $\mathbf{5 0}$ Normal Volunteers}

Concerning the tinnitus individuals, 10 (23.3\%) of them had elevated hearing thresholds within the mild to moderate hearing loss range, with 6 (13.9\%) of them being conductive loss, $3(7 \%)$ sensorineural, and 1 patient (2.3\%) had mixed

Table 1 Age and sex distribution of the participants

\begin{tabular}{|l|l|l|l|l|}
\hline \multirow{2}{*}{} & & $\begin{array}{l}\text { Study group } \\
\mathbf{N}=\mathbf{4 3}\end{array}$ & $\begin{array}{l}\text { Control group } \\
\boldsymbol{N}=\mathbf{5 0}\end{array}$ & $P$ value \\
\hline \multirow{3}{*}{ Age group } & $<25$ years & 3 & 6 & - \\
\cline { 2 - 5 } & $25-45$ years & 21 & 31 & - \\
\cline { 2 - 5 } & $>45$ years & 19 & 13 & - \\
\hline Mean age & Male & $40.9 \pm 11.7$ & $38.7 \pm 9.7$ & $\mathrm{t}(82)=1, p=0.3$ \\
\hline Sex & Female & 11 & 18 & $\left(x^{2}=1.2(d f=1) p=0.1\right)$ \\
\hline
\end{tabular}



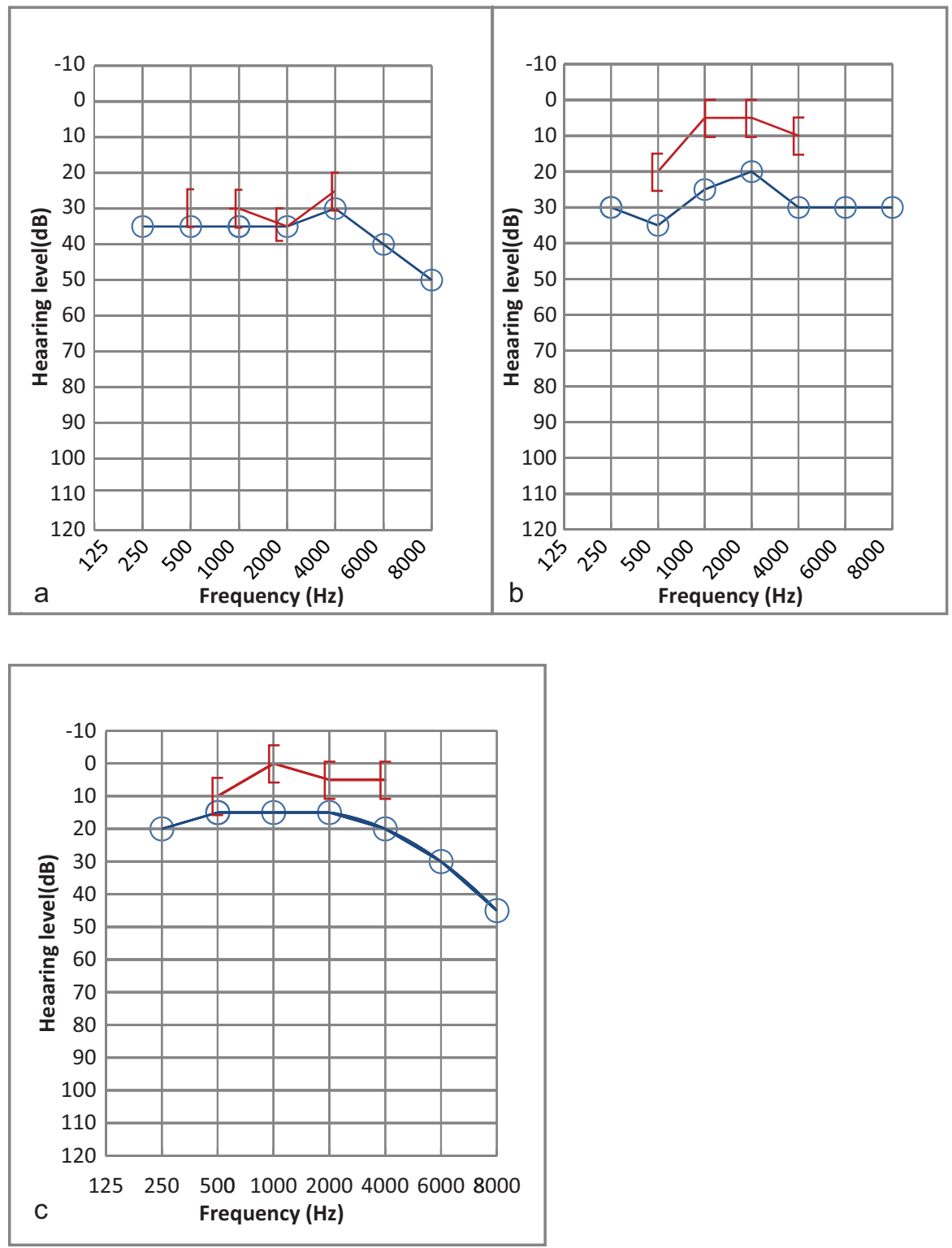

$$
\text { Keys: } \begin{aligned}
{[} & =\text { bone conduction right } \\
\bigcirc & =\text { air conduction right } \quad \times \quad \begin{array}{c}
\text { bone conduction left } \\
\text { air conduction left }
\end{array}
\end{aligned}
$$

Fig. 1 (a) Right audiogram of a 49-year-old female tinnitus sufferer in the study group with mild sensorineural hearing loss. (b) Right audiogram of a 53 -year-old female tinnitus sufferer in the study group with conductive hearing loss. (c) Right audiogram of a 38-year-old female tinnitus sufferer in the study group with high frequency hearing loss.

hearing loss. The elevated hearing thresholds were unilateral in 7 patients (16.3\%) and bilateral in 3 (7\%). All the 50 participants in the control group had normal hearing thresholds within the speech frequencies, but 12 of them had elevated hearing thresholds in the high frequencies ( 6 and $8 \mathrm{Khz}$ ), with $7(14 \%)$ of the individuals being unilateral whereas 5 of them (10\%) were bilateral (-Fig. 1a, 1b, 1c).

\section{Pure Tone Average (PTAv) of the Tinnitus Ears and Control Ears}

The 56 tinnitus ears had a mean PTAv of $14.8 \pm 9$ while that of the 100 control ears was $11.2 \pm 6$. The difference was significant $(U=2078 ; p=0.008)$. See - Table 2.
In the high frequencies, the 56 ears with tinnitus had a mean pure tone threshold of $20.9 \mathrm{dBHL}$ at $6 \mathrm{KHz}, 19.1 \mathrm{dBHL}$ at $8 \mathrm{KHz}$, while the 100 control ears without tinnitus had a mean pure tone threshold of $16.6 \mathrm{dBHL}$ at $6 \mathrm{KHz}, 15 \mathrm{dBHL}$ at $8 \mathrm{KHz}$. These differences were not significant $(U=2366$, $p=0.05$ )

\section{Comparison of the Tinnitus Ears and the Non-Tinnitus Ears in Participants with Unilateral Tinnitus}

The PTAv for the 30 ears with tinnitus was $17 \pm 10.4$, while the PTAv of the 30 contralateral ears without tinnitus was $15.7 \pm 13.2$. The difference was not significant $(U=448$; $p=0.5)$. 
Table 2 The mean pure tone thresholds for the 56 ears with tinnitus among the study group and the 100 control ears

\begin{tabular}{|l|l|l|}
\hline Frequency $(\mathrm{Hz})$ & $\begin{array}{l}\text { Study ears } \\
(\boldsymbol{n}=\mathbf{5 6})\end{array}$ & $\begin{array}{l}\text { Control ears } \\
(\boldsymbol{n}=\mathbf{1 0 0})\end{array}$ \\
\hline 500 & 17 & 11.2 \\
\hline 1000 & 14.4 & 11 \\
\hline 2000 & 12.8 & 10.3 \\
\hline 4000 & 14.4 & 12.5 \\
\hline
\end{tabular}

\section{Comparison of the Contralateral Non-Tinnitus Ears and the Control Ears}

The PTAv in the 100 ears of the control group without tinnitus was significantly better than the PTAv of the 30 contralateral non-tinnitus ears among the participants with unilateral tinnitus ( $\mathrm{U}=1136 ; p=0.02)$.

\section{Discussion}

\section{Pure Tone Average}

In this study, 10 (23.3\%) individuals with tinnitus were observed to have hearing loss as measured by pure tone audiometry, despite the fact that all of them reported that they had normal hearing. This implies that the hearing impairment was mild enough to have gone unnoticed by the participants or that the participants may have blamed the tinnitus for their difficulties with hearing. The subjects with unilateral tinnitus may also have been compensating with the contralateral ear without tinnitus.

Comparison between the 56 ears affected by tinnitus and the 100 ears not affected by tinnitus showed that the ears affected by tinnitus had a higher mean PTAv, which was significantly worse than the mean pure tone average of the ears not affected by tinnitus, this indicates that ears with tinnitus are likely to have elevated pure tone thresholds and hearing loss, even in tinnitus sufferers with self-reported normal hearing.

Several other studies made observations similar to those in this study. $8,9,11,15,16$ Their results indicated that patients with hearing loss are likely to present with tinnitus as their main complaints while majority of tinnitus sufferers may have some degree of hearing loss..$^{8,9,11,15,16}$ Monzani et al observed that 74 of the 100 tinnitus sufferers in their study had hearing loss. ${ }^{9}$ Martinesi et al observed that $64.14 \%$ of the 312 tinnitus sufferers in their study had varying degrees of hearing loss. ${ }^{17}$ However, none of these studies focused on determining the hearing thresholds of tinnitus patients with self-perceived normal hearing and none compared them to individuals without tinnitus. The elevated mean PTAv observed among the tinnitus sufferers in this study and in the 56 ears with tinnitus indicate that patients with tinnitus are more likely to have hearing loss than those without tinnitus. Therefore audiometry should be routinely performed for all patients presenting with tinnitus regardless of their self-reported hearing status. None of the subjects in the control group had PTAv greater than $25 \mathrm{dBHL}$ in the speech frequencies.

\section{Comparison of the PTAv between Tinnitus and Non- Tinnitus Ears in Subjects with Unilateral Tinnitus}

For the subjects with unilateral tinnitus, the mean pure tone average in the ears with tinnitus was higher than that in the contralateral ears without tinnitus but this was not statistically significant. Park et al made similar observations when they compared the pure tone thresholds in the ears of patients with unilateral tinnitus and did not observe a significant difference between the ears with tinnitus and their contralateral ears without tinnitus. However, with the use of Distortion Product Otoacoustic Emissions (DPOAE), a significant decrease in DPOAE threshold was observed in the ears with tinnitus. ${ }^{14}$ In studies that relied on otoacoustic emissions to assess tinnitus sufferers, authors observed that a significant proportion of the tinnitus ears with normal pure tone thresholds had defective otoacoustic emissions, which indicates that some patients with tinnitus may have abnormal outer hair cell function despite a normal pure tone average. $^{14,18,19}$

\section{Comparison of the Contralateral Non-Tinnitus Ears and the Control Ears}

In this study, we observed that the subjects with unilateral tinnitus had a significantly worse mean PTAv $(15.7 \pm 13.2)$ in the 30 contralateral ears without tinnitus than the mean PTAv $(11.2 \pm 5.3)$ in the 100 ears of the control group without tinnitus. This indicates that patients with tinnitus are likely to have elevated pure tone thresholds and that this elevated pure tone thresholds are likely to be seen in the contralateral unaffected ears of those with unilateral tinnitus. It appears that the contralateral unaffected ears of the subjects with unilateral tinnitus may have been exposed to the same etiological factor as the ear with tinnitus and may give rise to tinnitus in the future. It could also indicate that the insult to the contralateral ears without tinnitus was not severe enough to give rise to tinnitus. There is paucity of data on the hearing threshold of the contralateral non-tinnitus ear of the unilateral tinnitus sufferer and how it compares to the hearing threshold of healthy individuals without tinnitus. This information will help in the counselling of patients with unilateral tinnitus and will contribute to better patient management.

\section{Type of Hearing Loss}

In this study, out of the 10 (23.3\%) subjects with hearing loss, conductive hearing loss was the commonest type of hearing loss observed in 6 (13.9\%), while sensorineural hearing loss was observed in 3 (7\%) of the participants and mixed hearing loss in 1 (2.3\%). Our results defer from the findings in a study by Martines et al where sensorineural hearing loss was seen in $74.6 \%$ of tinnitus sufferers, mixed hearing loss in $14.7 \%$, and conductive hearing loss in $10.7 \% .^{3}$ Monzani et al also observed that sensorineural hearing loss was the commonest hearing loss amongst the tinnitus sufferers in their study. ${ }^{9}$ The conductive hearing loss observed in these subjects may be attributed to some subclinical middle ear disease such as Eustachian tube dysfunction which implies that these subjects are likely to have some improvement with therapy. The results of further middle ear analysis unfortunately are not available for this research. 
Despite having a normal PTAv, 10 subjects (23.3\%) of the study group had worsening hearing thresholds in their high frequency ranges, a fact not taken into account while calculating their standard pure tone hearing thresholds. Similar observations were made in the studies by Martines et al and Monzani et al, where they calculated the threshold in the high frequencies of $4 \mathrm{kHz}, 6 \mathrm{kHz}$, and $8 \mathrm{kHz} .{ }^{3,9}$ However both studies did not make use of a control group and, therefore, were unable to compare the high frequency hearing thresholds of the tinnitus sufferers to that of healthy controls. When the mean high frequency PTAv of the 56 ears affected by tinnitus was compared with the 100 ears of healthy controls, we observed that tinnitus sufferers had higher mean high frequency PTAv than the control group, but this difference was not significant.

This finding implies that high frequency hearing loss is likely to be present in tinnitus sufferers and in those without tinnitus, although tinnitus sufferers may have slightly worse high frequency thresholds.

\section{Conclusion}

Tinnitus sufferers with self-professed normal hearing are more likely to have hearing loss than none sufferers; however, high frequency hearing loss is likely to be observed in the tinnitus sufferer and in those without tinnitus.

The pure tone audiometric thresholds of the unaffected non-tinnitus ear in those with unilateral tinnitus is likely to be elevated when compared with that of individuals without tinnitus, indicating that these may also have a similar pathology as the tinnitus ear.

Tinnitus sufferers should therefore undergo routine audiometric evaluation even in cases where they report normal hearing.

\section{Conflict of Interest}

There is no conflict of interest to declare and no external source of funding used in the research.

\section{References}

1 Axelsson A, Ringdahl A. Tinnitus-a study of its prevalence and characteristics. Br J Audiol 1989;23(1):53-62

2 Aaron GB, Wayne KR. Inner ear: tinnitus. Emed 2013
3 Martines F, Bentivegna D, Martines E, Sciacca V, Martinciglio G. Assessing audiological, pathophysiological and psychological variables in tinnitus patients with or without hearing loss. Eur Arch Otorhinolaryngol 2010;267(11):1685-1693

4 Frank M, Marie BT, Barry L, et al. Auditory hallucinations: An audiological perspective. Hearing J 2007;60:32-52

5 Santos RMR, Sanchez TG, Bento RF, Lucia MC. Auditory hallucinations in tinnitus patients: Emotional relationships and depression. Int Arch Otorhinolaryngol 2012;16(3):322-327

6 Cima R, Joore M, Maes I, et al. Cost-effectiveness of multidisciplinary management of Tinnitus at a specialized Tinnitus centre. BMC Health Serv Res 2009;9:29

7 Ogunleye AO, Labaran AO. Presbycusis in Nigerians at the University College Hospital, Ibadan. Afr J Med Med Sci 2005;34(3): 293-296

8 Adobamen PO, Ogisi FO. Symptoms Associated With Hearing Loss As Seen In the University Of Benin Teaching Hospital, Benin City, Nigeria. Gomal J Med Sci 2011;9:159-162

9 Monzani D, Genovese E, Marrara A, et al. Validity of the Italian adaptation of the Tinnitus Handicap Inventory; focus on quality of life and psychological distress in tinnitus-sufferers. Acta Otorhinolaryngol Ital 2008;28(3):126-134

10 Snow JB. Tinnitus: theory and management. Hamilton, London: BC Decker; 2004:16-63

11 Schlee W, Kleinjung T, Hiller W, Goebel G, Kolassa IT, Langguth B. Does tinnitus distress depend on age of onset? PLoS ONE 2011; 6(11):e27379

12 Bauer CA. Mechanisms of tinnitus generation. Curr Opin Otolaryngol Head Neck Surg 2004;12(5):413-417

13 World Health Organization. Prevention of Blindness and deafness: Grades of HearingImpairment. http://www.who.int/pbd/deafness/hearing_impairment_grades/en/index.html2002(accessed 17th April 2012).

14 Park JP, Lim HW, Shim BS, et al. Interaural differences of distortion product otoacoustic emission amplitudes in patients with unilateral tinnitus. Otolaryngol Head Neck Surg 2013;148(3):456-459

15 Gopinath B, McMahon CM, Rochtchina E, Karpa MJ, Mitchell P. Incidence, persistence, and progression of tinnitus symptoms in older adults: the Blue Mountains Hearing Study. Ear Hear 2010; 31(3):407-412

16 Shargorodsky J, Curhan GC, Farwell WR. Prevalence and characteristics of tinnitus among US adults. Am J Med 2010;123(8):711-718

17 Martines F, Bentivegna D, Di Piazza F, Martines E, Sciacca V, Martinciglio G. Investigation of Tinnitus Patients in Italy: Clinical and Audiological Characteristics. Int J Otolaryngol 2010;20(10): 265861

18 Granjeiro RC, Kehrle HM, de Oliveira TS, Sampaio ALL, de Oliveira CA. Is the degree of discomfort caused by tinnitus in normalhearing individuals correlated with psychiatric disorders? Otolaryngol Head Neck Surg 2013;148(4):658-663

19 Ami M, Abdullah A, Awang MA, Liyab B, Saim L. Relation of distortion product otoacoustic emission with tinnitus. Laryngoscope 2008;118(4):712-717 